\title{
Structure of Vertical Wind Profiles
}

\author{
By \\ Elmar R. Reiter \\ Department of Atmospheric Science \\ Colorado State University \\ Fort Collins, Colorado
}

\section{Department of \\ Atmospheric Science}

\author{
Paper No. 156
}




\title{
Structure of vertical wind profiles
}

\author{
Elmar R. Reiter \\ Department of Atmospheric Science \\ Colorado State University, Fort Collins, Colorado 80521
}

(Received August 6, 1969.)

\begin{abstract}
An exponential relationship between the vertical wind shear and the layer thickness over which this shear is measured has been derived. From an analysis of the Richardson number it appears that shallow layers reaching maximum shearing conditions under the established power law should be turbulent, irrespective of the prevailing thermal stability conditions.
\end{abstract}

\section{TURBULENCE INFORMATION FROM VERTICAL WIND PROFILES}

It has been shown by Essenwanger [1963, 1967] that vertical wind shears measured by detailed wind soundings, such as the FPS-16 tracks of 'Jimspheres,' yield exponential relationships of the form

$$
\langle\Delta v\rangle=a_{0}(\Delta h)^{a_{1}}
$$

where $\langle\Delta v\rangle$ denotes the mean total shear in meters per second (measured as the velocity vector difference over the height increment $\Delta h), a_{0}$ is a constant, and $a_{1}$ is the exponent of the relationship in question. (For further details, see Essenwanger and Reiter [1969]). The following exponents $a_{1}$ have been found from vertical wind soundings:

$\begin{array}{ll}\text { Mean wind shears } & a_{1}=1 / 2 \\ \text { Mean extreme shears } & a_{1}=1 / 3 \\ \text { Transient (nonstationary) fluctuations } & a_{1}=0 \\ \text { Persistent mesoscale } & a_{1}=4 / 5\end{array}$

A transverse structure function may be defined as

$$
D_{\imath}(\mathrm{r})=\overline{\left[v_{t}\left(\mathrm{r}+\mathrm{r}_{1}\right)-v_{\imath}\left(\mathrm{r}_{1}\right)\right]^{2}}
$$

where $v_{t}$ is the velocity component transverse to the distance vector $\mathrm{r}_{1}$ [Tatarski, 1961]. For $\mathrm{r}_{1} \equiv h$ and $r=\Delta h$, it is easily seen that (1) relates to (2) through the expression

$$
\sigma_{\Delta \Delta}^{2}=-\langle\Delta v\rangle^{2}+D_{u}(\mathrm{r})
$$

where $\sigma_{\Delta L}$ is the standard deviation of the shears $\Delta v$ measured over the height incremental $\Delta h$.

It has been shown by Tatarski [1961] that for the structure function

$$
D(r)=c^{2} r \quad \text { for } \quad 0<p<2
$$

Copyright 1969 by the American Geophysical Union. the one-dimensional spectral density function

$$
E(k)=\frac{\Gamma(p+1)}{2 \pi} \sin \frac{\pi p}{2} c^{2} k^{b}
$$

holds, where

$$
b=-(p+1)
$$

From the foregoing it may be seen that $a_{1}=p$, From this it appears that the exponential reli ship for mean extreme shears leads to a ' $-5 / 3$ ' trum slope.

According to Kolmogorov [1941] and $\mathrm{Obu}$ [1941], conditions in the inertial subrange are acterized by

$$
D_{t \imath}(\mathrm{r})=\frac{4}{3} C \cdot(\epsilon r)^{2 / 3} \text { for } \quad l_{0} \ll r \ll L
$$

where $l_{0}$ and $L$ characterize the inner and the 1 scales of turbulence, respectively, and where $C$ constant of the order of unity.

$$
a_{0}=\left(\frac{4}{3} C\right)^{1 / 2} \cdot \epsilon^{1 / 3}
$$

Figure 1 contains the data sample of mean treme shears obtained by Essenwanger [1963] tween the ground level and $30 \mathrm{~km}$. Taking $a_{1}=$ we may evaluate the coefficient as $a_{0}=1.2$ units of $\mathrm{m}^{2 / 3} \mathrm{sec}^{-1}$.

As a matter of interest, it should be pointed that Vinnichenko and Dutton [1969] found th ' $-5 / 3$ ' slope prevails in the horizontal compor of perturbation motion from the inertial subr: through the synoptic scale. The vertical structur the atmosphere, according to the preceding dis sion, seems to align itself in the same ' $-5 / 3$ ' ex] ential relationship of horizontal motions, if one siders the extreme velocity variations only. How the vertical structuring shows considerably 


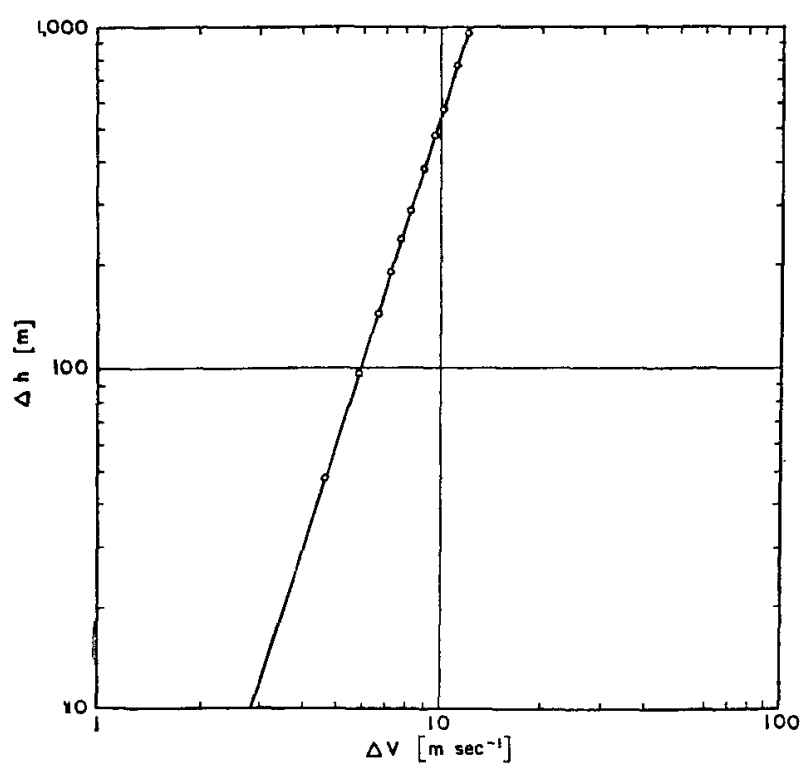

Fig. 1. Wind vector difference $\Delta V\left(\mathrm{~m} \mathrm{sec}^{-1}\right)$ measured over height increment $\Delta h(m)$ on logarithmic scale. [From Essenwanger, 1963.]

shear than does the horizontal mesoscale. This fact is apparent even from a comparison of the mean large-scale vertical and the horizontal shears around a jet stream.

\section{RICHARDSON NUMBER}

It has been pointed out by Reiter and Lester $[1967,1968]$ that the Richardson numbers evaluated from measurements in the free atmosphere, by virtue of the presence of a pronounced mesoscale in vertical wind profiles, reveal a certain dependence on the scale length $\Delta h$ over which wind shear and thermal stability are evaluated. From (1) and from the observational evidence supporting it, we have seen that mean extreme wind shears themselves are scaledependent. We are not aware of any corresponding statistics for the vertical gradient of temperature or potential temperature. Recent developments in smalllag temperature sensors [Caplan and Camp, 1968] may, however, allow such an evaluation in the near future.

We will assume that the statistical relationship between mean maximum vertical shear and layer thickness (shown in Figure 1) is brought about by the onset of turbulence that prohibits the development of excessive shears. Using the scale dependence of these maximum shears expressed by (1) and by an exponent $a_{1}=1 / 3$, in the Richardson number, we obtain

$$
R i=\frac{(g / \theta) \Delta \theta / \Delta h}{(\Delta V / \Delta h)^{2}}
$$

and substituting for $\Delta v$ from (1) (with $a_{1}=1 / 3$ ), we arrive at

$$
R i=g \cdot \Delta \theta \cdot \Delta h^{1 / 3} / \theta \cdot a_{0}{ }^{2}
$$

Businger [1968] argues that turbulence will develop out of shear flow when the Richardson number is below a critical level of 0.25 . Let us assume that this value of $R i$ also characterizes the critical limit to which the mean maximum wind shears in expression (9) may develop. With this assumption, we arrive at

$$
\Delta \theta=11.77 /(\Delta h)^{1 / 3}
$$

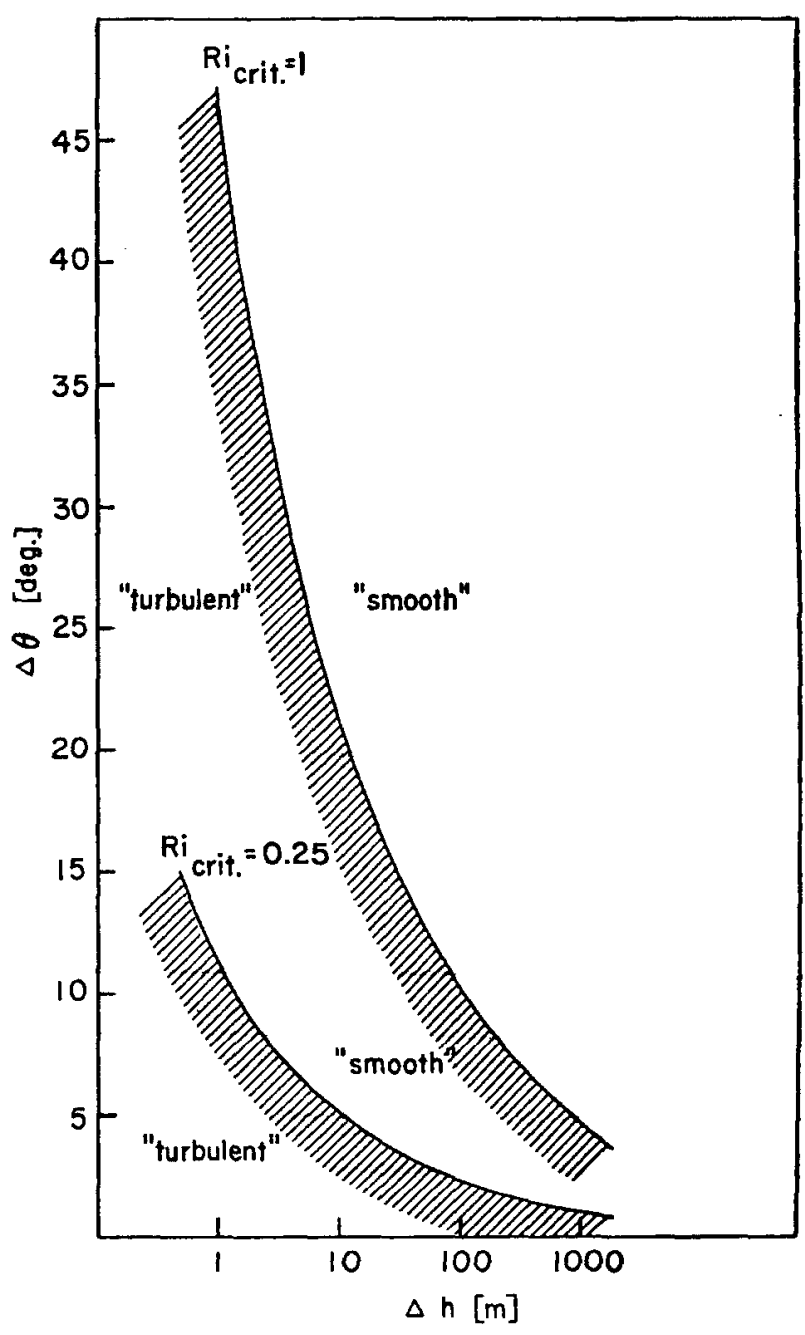

Fig. 2. Maximum vertical temperature differences $\Delta \theta$ in degrees under conditions of maximum vertical wind shear as given by equation $1\left(a_{0}=1.22 \mathrm{~m}^{2 / 3} \mathrm{sec}^{-2}, a_{1}=1 / 3\right)$ for 'critical' Richardson numbers as indicated. 
where $\theta=310^{\circ}, g=9.8 \mathrm{~m} \mathrm{sec}^{-2}$, and $a_{0}=1.22$ $\mathrm{m}^{2 / 3} \sec ^{-1}$. A critical Richardson number of 1 would yield, for the same parameter values as above,

$$
\Delta \theta=47.08 /(\Delta h)^{1 / 3}
$$

where $\Delta \theta$ indicates the maximum vertical potential temperature gradient as a function of layer thickness $\Delta h$, which would be required to maintain a state of 'just no turbulence' under (mean) extreme shear conditions. The two functional relationships (10) and (11) are shown in Figure 2.

If for a given height increment $\Delta h$, the observed temperature difference falls below the lines shown in Figure 2, turbulence should be expected, provided that the corresponding wind shear in this layer reached maximum values. From Figure 3 it appears that strong inversion conditions of the order of $12 \% / 1$ meter, or $5 \% / 10$ meters should rarely occur in the atmosphere. We may conclude, therefo: shallow layers revealing maximum shear con should always ensue in turbulent flow. Deep however, may be turbulent or 'smooth,' even the wind shear conditions given by (1) with $a_{1}$ depending on the actual temperature structure der isothermal conditions in the stratospher $\Delta \theta \sim 10^{\circ}$ for $\Delta h=1000$ meter. Such a value still render 'smooth' conditions for $R i_{\text {erit }}=$ Turbulence may exist, however, for $R i_{\text {crit }}=1$

Reiter [1968], Reed [1968], and Businger I have speculated that the development of a turbulent region within a relatively thick sh layer should result in the development of an batic region with a nearly constant wind bo by two stable layers in which strong vertical shears are concentrated. The mixing pro within the turbulent layer also apply to passiv

Fig. 3. Temperature measurements over Cape Kennedy with Jimsphere sensor (dots) and rawinsonde (crosses) for dates as indicated. [After Caplan and Camp, 1968.]

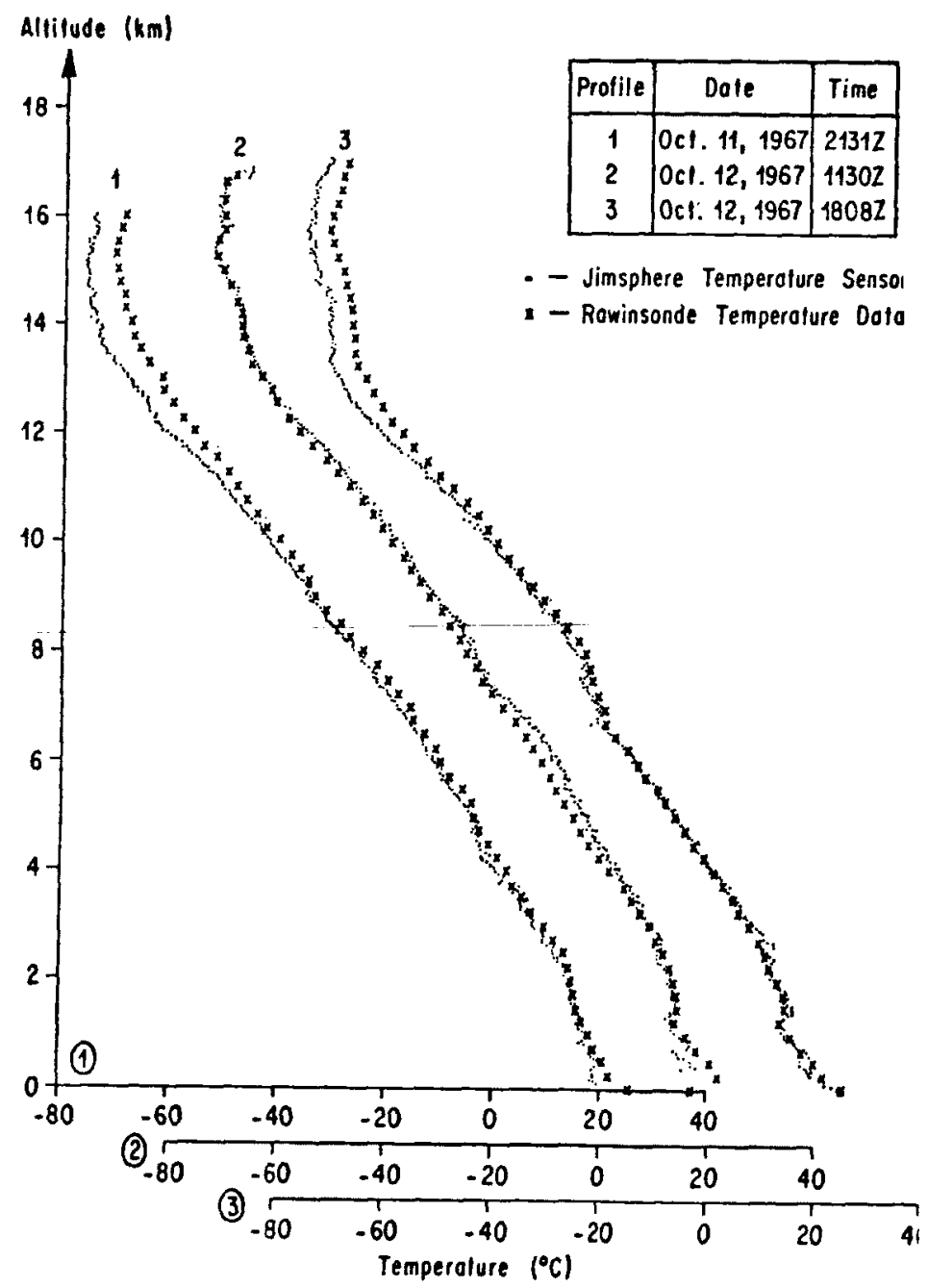


mixtures, such as water vapor. If a vertical gradient of this quantity existed prior to the onset of turbulence, the mixing process will 'concentrate' the gradient along the upper and lower boundaries of the turbulent layer. Backscattering of radio or radar signals will be enhanced by the resulting sharp gradients in the refractive index.

\section{REFERENCES}

Businger, J. A. (1968), On the energy supply of clear turbulence, Document D1-82-0740, Boeing Scientific Research Laboratory, Seattle, Wash.

Caplan, F. E., and D. W. Camp (1968), High resolution balloon-borne temperature sensor, in Proc. Nat. Conf. Aerospace Meteorol., New Orleans, pp. 76-78, American Meteorological Society, Boston, Mass.

Essenwanger, O. M. (1963), On the derivation of frequency distributions of vector shear values for small shear intervals, Geofis. Pura Appl., 56, 216-224.

Essenwanger, O. M. (1967), Comments on 'mesoscale structure of 11-20 km winds,' J. Appl. Meteorol., 6, 591593.

Essenwanger, O. M., and E. R. Reiter (1969), Power spectrum, structure function, vertical wind shear and turbu- lence in troposphere and stratosphere, (in English), Arch. Meteorol. Geophys. Bioklimatol., A, 18, 17-24.

Kolmogorov, A. N. (1941), Dissipation of energy in locally isotropic turbulence, (in Russian), Dokl. Akad. Nauk SSSR, 32,

Obukhov, A. M. (1941), On the distribution of energy in the spectrum of turbulent flow, (in Russian), Dokl. Akad. Nauk, SSSR, 32,

Reed, R. J. (1968), A study of the relation of clear air turbulence to the mesoscale structure of the jet stream region, Doc. D1-82-0740, Boeing Scientific Research Laboratories, Seattle, Wash.

Reiter, E. R. (1968), The nature of clear air turbulence: A review, Doc. D1-82-0740, Boeing Scientific Research Laboratories, Seattle, Wash.

Reiter, E. R., and P. F. Lester (1967), The dependence of the Richardson number on scale length, Atmos. Sci. Pap. 111, Colorado State University, Fort Collins.

Reiter, E. R., and P. F. Lester (1968), Richardson's number in the free atmosphere, Arch. Meteorol., Geophys. Bioklimatol., A, 17, 8-16.

Tatarski, V. I. (1961), Wave Propagation in a Turbulent Medium, McGraw-Hill, New York.

Vinnichenko, N. K., and T. A. Dutton (1969), Empirical studies of atmospheric structure and spectra in the free atmosphere, Radio Sci., 4(12), this issue. 